\title{
Performance of Parylene-Packaged Flexible Pentacene Thin-Film Transistors in Saline
}

\author{
Hsi-wen Lo and Yu-Chong Tai \\ Electrical Engineering, Div. of Engineering and Applied Science, \\ California Institute of Technology, Pasadena, CA, USA \\ California Institute of Technology, Electrical Engineering, M/C: 136-93, Pasadena, CA 91125, USA. \\ Tel: +1-626-395-3885, Fax: +1-626-584-9104, E-mail: lo@mems.caltech.edu
}

\begin{abstract}
A micro-fabricated parylene-packaged flexible pentacene thin film transistor is presented. Different from preceding devices that have been reported, this thin film transistor employs parylene as the substrate, the gate insulator and also the top protection layer. Also, this thin film transistor uses pentacene, an organic semiconductor with high mobility, as the active material. The fresh made thin film transistor shows a hole mobility of $0.022 \mathrm{~cm}^{2} / \mathrm{V}$-s. In spite of initial drops, the transistor's hole mobility stays at $0.001 \mathrm{~cm}^{2} / \mathrm{V}$-s after over 6-month soaking in saline. We can conclude that drifts in mobility from soaking do exist but they saturate to values still of promise In addition, we believe there's plenty of room to improve the parylene packaging such as by using thicker parylene (this work used only $1 \mu \mathrm{m}$ ).
\end{abstract}

Keywords: Pentacene, Parylene, Thin-film transistor

\section{INTRODUCTION}

Vision loss due to retinitis pigmentosa (RP) and age-related macular degeneration (AMD) has troubled millions of people around the world. Recently, a retinal prosthesis has been developed for the treatment of aged-related blindness. This technology is based on the concept of replacing photoreceptor function with an electronic device [1]. For this technology, a huge amount of electrodes are needed to achieve reasonable or high resolutions. A biocompatible and scalable high lead count electrode array for retinal prosthesis has been successfully fabricated[2]. These electrodes are directly connected to the implanted control electronics through metal interconnects. As the resolution increase, the number of electrodes and interconnects increase, too. So is the volume of the implanted device. One way to reduce the number of metal interconnects and to satisfy the small volume constraint is to introduce a multiplexer into the system. This multiplexer has to overcome such difficulties as the corrosive environment and integration with the metal interconnects and so on.

One revolutionary approach to solve the problem is to explore biocompatible electronics that do not require conventional hermetic packaging and, at the same time, flexible enough for implantation use.

The combination of organic semiconductor and polymer substrate can serve this purpose. Pentacene $\left(\mathrm{C}_{14} \mathrm{H}_{22}\right)$ thin film transistors (TFT) have been fabricated and possess a hole mobility up to $2.59 \mathrm{~cm}^{2} / \mathrm{V}-\mathrm{s}$ [3], which is comparable to the popular a-Si:H TFT technology.

Pentacene, however, is sensitive to oxygen so unprotected pentacene transistors are vulnerable to even normal environments. It is therefore interesting to use parylene (readily a proven biocompatible material) as a pentacene-protecting polymer. Parylene $\mathrm{C}$, a widely used MEMS (micro-electro-mechanical system) material, shows great flexibility (Young's modulus 4 GPA), chemical inertness and biocompatibility [4]. Parylene $\mathrm{C}$ has been recognized as a USP Class VI material and its intraocular biocompatibility has been studied [2] In fact, parylene has been and is being studied for both encapsulation layer [5] [6] and even as a new gate insulator. However, this work reports the first flexible parylene-pentacene electronics where parylene is exclusively used as the substrate, gate insulator and encapsulation layer.

\section{DEVICE FABRICATION}

Our parylene-packaged flexible pentacene TFTs used a bottom-contact configuration (Fig. 1) so as to avoid pentacene contamination from following processing. The basic technology involved in parylene-packaged pentacene TFT is the parylene/metal skin technology [7] [8]. The 
simplified fabrication process is illustrated in Fig. 2.

(a)

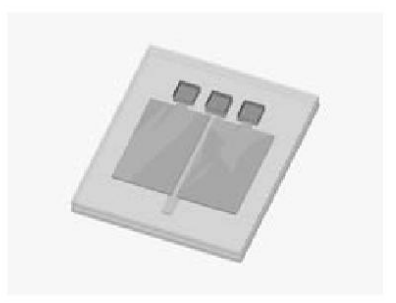

(b)

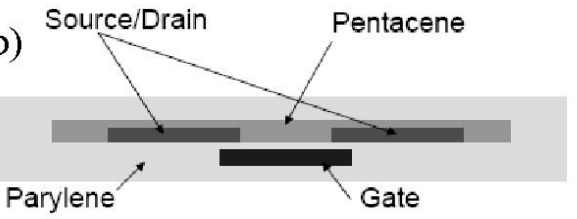

Fig 1 Isometric (a) and cross-section (b) views of bottom-contact configuration of parylene-packaged flexible pentacene TFTs

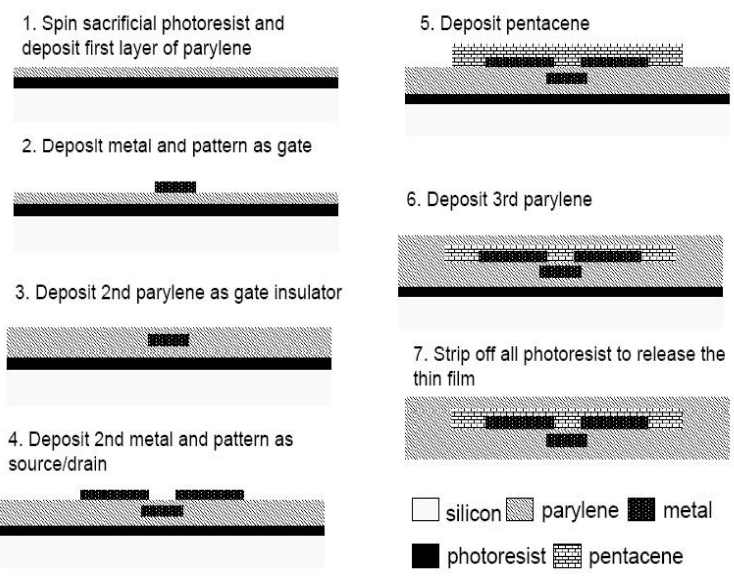

Fig 2 Simplified process flow

The fabrication started with photoresist-coated wafers. A $10-\mu \mathrm{m}$ parylene-C was first deposited as the substrate. A 1,500-angstron $\mathrm{Au}$ with 100 -angstron $\mathrm{Cr}$ was thermally deposited and patterned to be the gate. A $0.31-\mu \mathrm{m}$ parylene-C was then deposited as the gate dielectrics. Next, a 500-angstron $\mathrm{Au}$ was deposited and patterned as the sources and drain. Then, a 200-nm pentacene (as purchased from Sigma-Aldrich) was thermally-evaporated under high vacuum. A $1-\mu \mathrm{m}$ parylene-C was deposited as top-protecting layer. Finally, the whole parylene-pentacene TFTs was liftoff from the photoresist in a flexible MEMS form (Fig 3). (a)
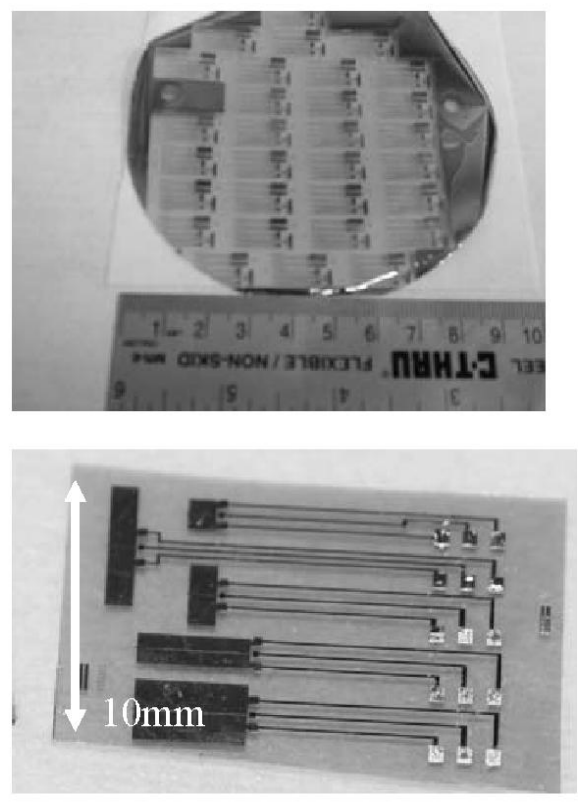

(b)

(c)

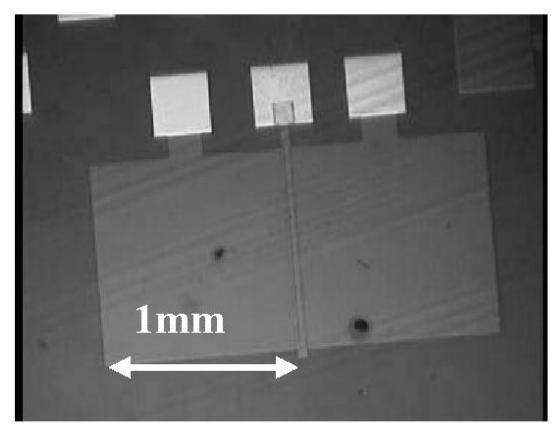

Fig 3 Fabricated Parylene film containing pentacene thin-film transistors. (a) Released film (b) Closer view (c) one of the transistors on the parylene film

\section{RESULTS}

We obtained the drain and gate characteristics of the thin-film transistor with a probe station and the HP4145B semiconductor parameter analyzer. The transistor was measured at room temperature. Fig 4 shows the drain and gate characteristics of an unsoaked thin-film transistor. 

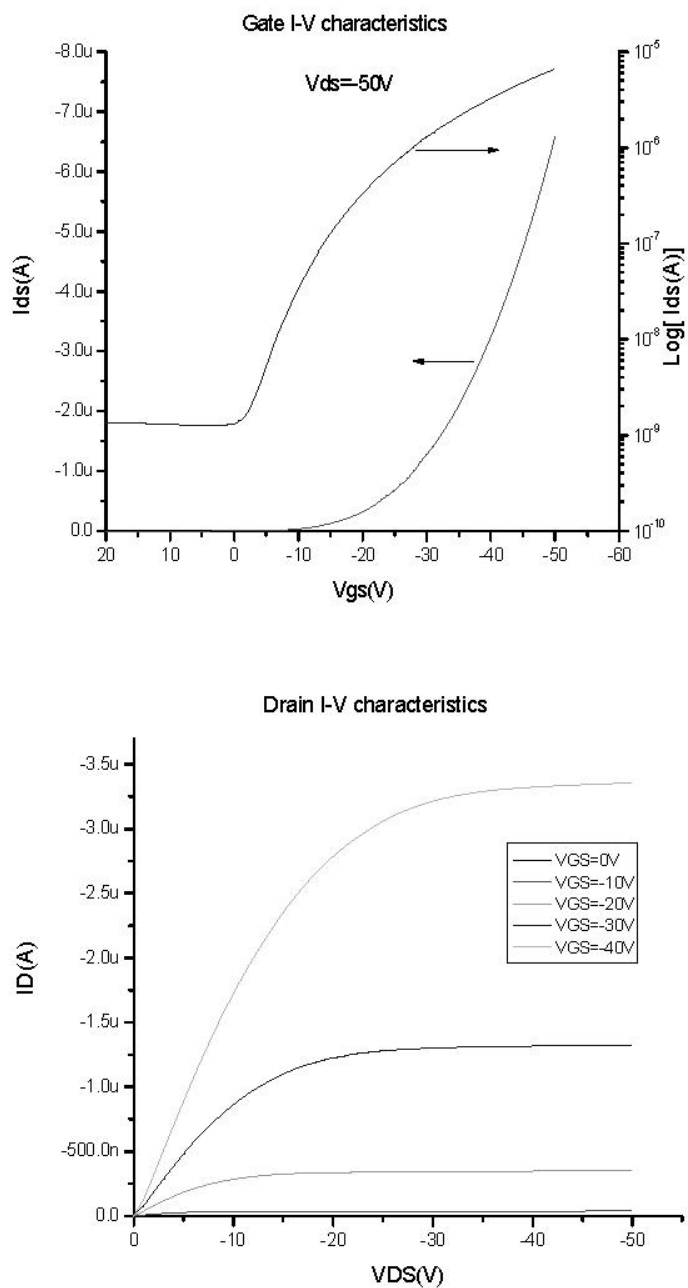

Fig 4 Drain (up) and Gate (down) I-V characteristics of pentacene thin-film transistors right after fabrication

The mobility of charge carrier $(\mu)$ in the saturation regime can be calculated from the drain current given by the equation.

$$
I_{D}=\frac{1}{2} \mu C_{i} \frac{W}{L}\left(V_{G S}-V_{T}\right)^{2}
$$

Take square root of both sides.

$$
\sqrt{I_{D}}=\sqrt{\frac{C_{i} W}{2 L}} \mu\left(V_{G S}-V_{T}\right)
$$

Solving this equation and use a definition of " $k$ " as

$$
\begin{gathered}
k=\sqrt{\frac{C_{i} W}{2 L} \mu} \\
\mu=\frac{2 L}{W C_{i}} k^{2}
\end{gathered}
$$

Equating the slope of the plot $\sqrt{I_{D}}$ versus $\mathrm{V}_{\mathrm{G}}$ to " $k$ " determines the $\mu$ in the saturation regime.

The unsoaked parylene-pentacene transistor shows a hole molibilty of $0.022 \mathrm{~cm}^{2} / \mathrm{V}$-s.

For bio-implantation applications, the parylene-pentacene flexible electronics must stand the bio-environment. To explore this, we soaked our parylene-pentacene thin-film transistors in saline at room temperature and monitored the hole mobility.

After a month's soaking, the mobilities from several transistors seem to always converge to $0.005 \mathrm{~cm}^{2} / \mathrm{V}$-s (Fig 5) indicating a saturated value but still manageable to make useful electronics. So far, soaking has been more than 6 months, and the pentacene hole mobilities stay at $0.001 \mathrm{~cm}^{2} / \mathrm{V}$-s (Fig 6)

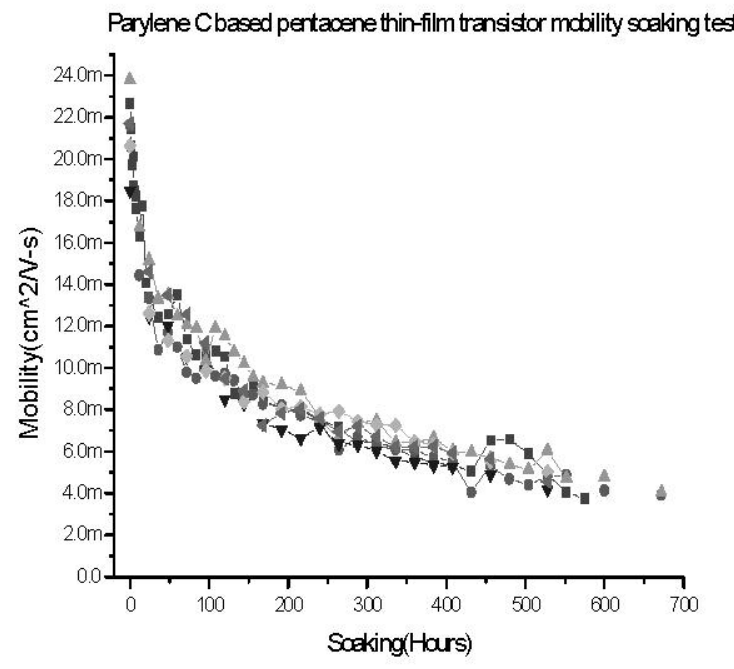

Fig5 Mobility versus time (1 month) 


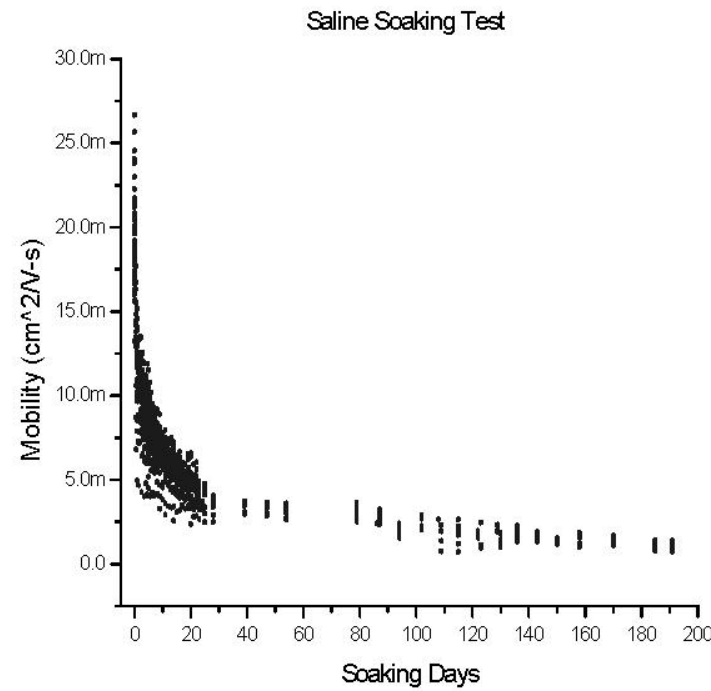

Fig 6 Mobility versus time (6 months)

Although continuous soaking is underway, we can conclude from the first data that the drifts in mobility from soaking do exist but they saturate to values still of promise. In addition, we believe there's plenty of room to improve the parylene packaging such as by using thicker parylene (this work used only $1 \mu \mathrm{m}$ ) or other techniques.

\section{ACKNOWLEDGEMENT}

The authors would like to thank Mr. Trevor Roper for his assistance with equipment and fabrication. We would also thank Tanya Owen, Christine Matsuki and other members of the Caltech Micromachining Laboratory for their assistance.

\section{REFERENCES}

1. Humayun, M.S., Intraocular retinal prosthesis. Tr: Am Ophth Soc, 2001. 99. 2. D. C. Rodger, J.D.W., M.S. Humayun, and Y.C. Tai. Scalable Flexible Chip-level Parylene Package For High Lead Count Retinal Prosthesis. in Transducer. 2005.

3. S.C. Lim, S.H.K., J.H. Lee, H.Y. Yu, Y. Park, D. Kim and T. Zyung, Organic thin-film transistors on plastic substrates. Elsevier Materials Science and Engineering B, 2005(121): p. 211-215.

4. Wolgemuth, L., Assessing the performance and suitability of parylene coating. Medical Device \& Diagnositic Industry, 2000. 22: p. 42-49.

5. I. Kymissis, C.D.D., and S.

Purushothanman, Patterning pentacene organic thin film transistors. Journal of Vacuum Science \& Technology, 2002. B(20(3)): p. 956-959.

6. D. Feili, M.S., T. Doerge, S. Kammer, and T. Stieglitz, Encapsulation of organic field effect transistors for flexible biomedical microimplants Elsevier Sensors and Actuators, 2005. A 120: p. 101-109.

7. M. Liger, N.P., Y.C. Tai, S. Ho and C.M. Ho. Large-area electrostatically-valved skins for adaptive flow control on ornithopter wings. in Technical Digest, Solid State Sensor and Actuator Workshop. 2002. Hilton Head Island, South Carolina, USA.

8. Y.C. Tai, F.J., Y. Xu, M. Liger, S. Ho and C.M. Ho. Flexible MEMS skins: technologies and applications. in Proceedings, Pacific Rim MEMS Workshop. 2002. Xiamen, China. 